\title{
William Pitt, les francs-maçons anglais et la loi sur les sociétés secrètes de 1799
}

\section{Pierre-Yves Beaurepaire}

\section{(2) OpenEdition \\ 1 Journals}

Édition électronique

URL : https://journals.openedition.org/ahrf/1935

DOI : 10.4000/ahrf.1935

ISSN : 1952-403X

\section{Éditeur :}

Armand Colin, Société des études robespierristes

\section{Édition imprimée}

Date de publication : 1 décembre 2005

Pagination : 185-194

ISSN : 0003-4436

\section{Référence électronique}

Pierre-Yves Beaurepaire, « William Pitt, les francs-maçons anglais et la loi sur les sociétés secrètes de 1799 », Annales historiques de la Révolution française [En ligne], 342 | octobre-décembre 2005, mis en ligne le 15 décembre 2008, consulté le 24 avril 2022. URL : http://journals.openedition.org/ahrf/1935 ; DOl : https://doi.org/10.4000/ahrf.1935

Ce document a été généré automatiquement le 24 avril 2022.

Tous droits réservés 


\title{
William Pitt, les francs-maçons anglais et la loi sur les sociétés secrètes de 1799
}

\author{
Pierre-Yves Beaurepaire
}

"C'est la franc-maçonnerie, mère et nourrice de toutes les sociétés secrètes depuis l'illuminisme bavarois auquel elle sert de souche, jusqu'à l'Internationale ou la Commune parisienne qui lui doit tous ses principes et tous ses forfaits ».

Nicolas Deschamps, Les Sociétés secrètes et la société ou philosophie de l'histoire contemporaine (1874-1876).

1 «Why are they persecuted? " titre le Literary Supplement du Times le 14 janvier 2000, à propos des francs-maçons britanniques. On se souvient de fait des débats qui ont enflammé la presse, le Parlement et le gouvernement britanniques à la fin des années 1990. Le Home Secretary (ministre de l'Intérieur) Jack Straw - depuis lors passé aux Affaires étrangères où il a pourfendu les options françaises lors de la deuxième guerre du Golfe- du cabinet de Tony Blair somme alors les francs-maçons fonctionnaires de justice et de police de déclarer leur appartenance à l'ordre, et les obédiences de faire connaître la liste de leurs membres, dans un contexte particulièrement difficile pour la franc-maçonnerie britannique. Vieillissement rapide, chute des effectifs maçonniques, rumeurs de corruption et de protection dont auraient profité des criminels francsmaçons de la part de juges et de policiers francs-maçons affectent profondément l'image d'une obédience revendiquant son appartenance à l'Establishment après avoir milité dès le $\mathrm{XVIII}^{\mathrm{e}}$ siècle pour l'obtention d'une charter of incorporation, une reconnaissance d'une utilité publique qui confine à l'institutionnalisation. Jack Straw s'est alors vu reprocher par l'un de ses propres collègues du gouvernement néotravailliste une intrusion inadmissible dans la vie privée et « de société ».

2 Cette méfiance de l'exécutif britannique et d'une fraction du Parlement à l'encontre de la franc-maçonnerie n'est pas nouvelle. Elle permet de faire retour sur un épisode critique de l'histoire maçonnique anglaise au cours de la tourmente révolutionnaire des années 1790, qui témoigne des difficultés récurrentes que rencontre l'ordre pour échapper à la confusion entre société à secrets et société secrète, entre les secrets ou 
mystères de l'initiation et le secret de la conspiration, y compris dans l'environnement apparemment le plus favorable. La prégnance des "discours de persécution " (René Girard) qui visent à expliquer le chaos révolutionnaire qui embrase l'Europe par le déchaînement d'une universelle conspiration associant protestants- en pays catholique - et papistes-, en terre protestante- républicains, philosophes, partisans des Lumières radicales, Illuminaten - les fameux Illuminés de Bavière de l'abbé Barruel-, sympathisants de la Révolution française, jacobins est frappante. Elle permet d'expliquer l'incompréhensible, comment l'Europe de l'Ancien Régime s'est effondrée, déchirée, par la "révélation ", celle du complot, dont on peut dès lors esquisser le scénario des préparatifs et l'entrée en scène des conjurés. On sait que de tels discours mobilisant irrationnel et mentalité obsidionale sont à l'œuvre tout au long des XIX et $\mathrm{xx}^{\mathrm{e}}$ siècles, pour expliquer aussi bien le Printemps des peuples de 1848 que l'effondrement de l'Empire russe en 1917, de la France en 1940 ou de l'Empire soviétique après 1989. L'Angleterre des années 1790 n'est pas restée à l'abri des impacts de ces discours de persécution stigmatisant le secret des francs-maçons comme éminemment subversif et suspect - «discriminant féroce» (Sophie Wahnich) par excellence de la période révolutionnaire.

3 Quelques mois après l'entrée en guerre de la République française contre la GrandeBretagne, le Sentimental and Masonic Magazine de juillet 1793 publie à Dublin «l'Adresse à Sa Majesté de la Grande Loge de l'ancienne fraternité des francs et acceptés maçons, faite par un homme de condition et de haut rang dans le monde public ", savoir le major-général comte de Moira, marquis de Hastings, Acting Grand Master de la Grande Loge d'Angleterre (Modernes), victorieux du général Pichegru à Bruges où il secourt le duc d'York (fils du roi George III et franc-maçon) encerclé par les troupes françaises :

"Il est écrit, Sire, dans les fondements de notre ordre, que nous ne nous livrerons pas durant nos assemblées à des discussions religieuses ou politiques : en effet notre ordre fraternel étant composé d'hommes de nations diverses, professant des doctrines religieuses différentes, et attachés à des systèmes de gouvernements opposés, de telles discussions, qui dressent les esprits les uns contre les autres, pourraient offenser ou semer la désunion parmi les frères. Cependant, une crise aussi imprévisible que la crise actuelle, justifie selon nous une exception à cette règle; notre premier devoir en tant que Britanniques l'emportant sur toute autre considération, nous joignons, sans plus tarder, notre voix à celle de nos concitoyens pour proclamer l'attachement fervent, que nous éprouvons tous à l'égard du gouvernement assuré par le roi, la chambre des Lords et la Chambre des Communes, établi par la Glorieuse Révolution de 1688 ».

4 La Grande Loge ne manque aucune occasion de réaffirmer son loyalisme à l'égard de la dynastie hanovrienne et par là même de mettre en scène son indéfectible appartenance à l'Establishment, puisque six des sept fils de George III sont francs-maçons. Elle félicite ainsi le prince de Galles, qui n'est autre que son Grand Maître, à l'occasion de son mariage en 1795. En 1800, à l'occasion de l'attentat manqué contre George III au théâtre de Drury Lane, les deux obédiences, Modernes - celle de 1717- et Anciens - celle de 1751font assaut de témoignages horrifiés contre l'»atroce attentat» et de serments d'allégeance sans faille. Mais, les francs-maçons britanniques savent aussi jouer de la presse maçonnique; le Sentimental and Masonic Magazine, qui paraît à Dublin en 1792-1795, consacre l'essentiel de ses colonnes à la franc-maçonnerie anglaise des Modernes et à des rubriques d'informations générales, car le journal ne vise pas seulement le public maçon; quant au Freemason's magazine, il est publié à Londres (devenu en 1797 The Scientific Magazine and Freemason's Repository- le Magazine 
scientifique et répertoire du franc-maçon-) pour riposter aux attaques des anti-maçons. Le comte de Moira lui-même monte au créneau pour dénoncer les calomnies de l'abbé Barruel - qui avait pourtant pris soin de ménager les francs-maçons lorsqu'il était l'hôte d'Edmund Burke- et de John Robison (1739-1805), ancien franc-maçon, professeur d'histoire naturelle à l'Université d'Édimbourg, secrétaire-général de la Royal Society, auteur des fameuses Proofs of a Conspiracy against all the religions and governments of Europe, Carried on in the secret meetings of Freemasons, Illuminati and Reading societies, collected from good authorities (Preuves d'une conspiration contre toutes les religions et les gouvernements d'Europe fomentée dans les assemblées secrètes des francs-maçons, illuminés et les sociétés de lecture, obtenues de bonnes sources) en 1797.

5 Pourtant, si elle considère que tout maçon doit être loyal à son souverain au nom de l'article II des Constitutions, la Grande Loge cherche à rester en dehors du combat politique. Lorsqu'elle réagit à des événements dramatiques comme l'attentat du 15 mai 1800, elle éprouve la nécessité de justifier sa prise de parole. Si le Sentimental and Masonic Magazine consacre des articles à Edmund Burke, auteur des Réflexions sur la révolution de France, où il condamne sans appel la Révolution française, la Grande Loge ne fait jamais référence à son appartenance à l'ordre - Burke est membre de la Jerusalem Lodge $n^{\circ} 44$, orient de Londres- pour illustrer l'hostilité des francs-maçons anglais aux idées subversives. Le caractère bouillant et polémique de Burke dérange manifestement. En revanche, Anciens et Modernes prennent soin de préciser que l'égalité maçonnique n'a rien à voir avec l'égalitarisme révolutionnaire. Le Freemason's Magazine publie en août 1794 cette mise au point: "L'égalité qui règne parmi les maçons est une condescendance temporaire et volontaire de supérieurs à l'égard d'inférieurs pendant les assemblées de loge (et pas plus longtemps) dans le dessein louable de promouvoir l'un des grands principes de l'ordre, l'amour fraternel. Lorsque l'assemblée est terminée, chacun retrouve sa place dans la société et honneur est rendu à qui de droit ». La presse maçonnique narre également le martyre de Louis XVI et de sa famille, et le Sentimental and Masonic Magazine publie une gravure du roi faisant le signe de détresse maçonnique devant l'échafaud. Une certaine publicité est accordée aux démonstrations de patriotisme des loges, ainsi lorsque la loge Red Lion Street à Wapping envisage la création d'une association d'apprentissage au maniement des armes pour parer à tout débarquement français, ou lorsque la loge de l'Unanimité (Lodge of Unanimity), orient de Wakefield, lance une contribution pour l'effort de guerre sur le ton de haine à toutes les révolutions. Mais, manifestement, les dirigeants craignent les débordements que de tels assauts maladroits de patriotisme pourraient causer.

$6 \quad$ L'attitude des obédiences britanniques est donc globalement défensive. Elles cherchent surtout à rassurer le pouvoir et à parer les coups des antimaçons. De son côté, la Grande Loge d'Irlande réitère l'interdiction des débats politiques, et veille à ne pas donner prise aux critiques de certains détracteurs qui dénoncent la présence en loges de membres des United Irishmen (" Société unie de la nation irlandaise visant à faire de tous les Irlandais des citoyens et de tous les citoyens des Irlandais ", créée en 1791). Le Grand Maître Lord Donoughmore prend lui-même la tête d'un corps de Masonic Volunteers lorsque la menace d'un débarquement français se précise. L'importance de la Société des United Irishmen, son influence en Grande-Bretagne que traduit la création des United Scotsmen et United Englishmen, la présence de nombreux ouvriers irlandais dans l'Angleterre industrielle séduits par l'émancipation nationale et l'exigence de réformes sociales et politiques radicales, l'envoi d'émissaires en France, puis le 
débarquement de troupes françaises, alimentent clairement la psychose des associations subversives et des conspirations révolutionnaires, que ne réduisent $\mathrm{ni}$ l'échec de l'insurrection irlandaise ni celui de l'intervention militaire française.

Replacée dans l'atmosphère délétère ambiante, reliée aux rumeurs d'infiltration des loges par les Illuminaten - Robison croit pouvoir dénombrer huit loges d'» Illuminati » en Grande-Bretagne dans son ouvrage de 1797-, par les « jacobins » et les United Irishmen, la situation des Grandes Loges britanniques est donc en réalité particulièrement délicate. La menace de l'amalgame plane en permanence, renforcée par les informations parvenues au gouvernement de William Pitt ou au Secret Committee de la Chambre des Communes sur l'existence d'un serment de fidélité et de secret prêté par les United Irishmen, la pratique de rituels et de catéchismes dont les emprunts maçonniques, fussent-ils formels, ne sont pas discutables, ainsi que d'une organisation solidement hiérarchisée et cloisonnée à travers le pays - la réalité est cependant beaucoup plus modeste. Il est donc important d'étudier de près les étapes de préparation de la Unlawful Societies Act du 12 juillet 1799 dont le titre exact et tout à fait révélateur - «Loi pour la suppression radicale (most effectual suppression) des sociétés établies dans le but de sédition et trahison, et pour une meilleure prévention des pratiques de trahison et de sédition »- pour saisir la manière dont les francs-maçons sont perçus, exposés ou au contraire innocentés. L'enjeu est essentiel, tant du point de vue de la survie de la franc-maçonnerie en Grande-Bretagne, que de la remise en cause de sa relation intime avec la monarchie et l'Establishment. En outre, on ignore le plus souvent que les dispositions de la loi s'appliquent jusqu'à la vaste réforme judiciaire entérinée par le Criminal Justice Act en... 1967.

Un premier ensemble de textes avait déjà été voté, notamment le Unlawful Oaths Act de 1797, loi sur les serments illégaux, qui avait épargné les francs-maçons mais sérieusement affaibli les sociétés politiques radicales comme la London Corresponding Society, avec pour risque - effectivement concrétisé- de pousser ses membres les plus déterminés dans l'engrenage d'un engagement clandestin et d'une relation toujours plus poussée avec les mouvements républicains irlandais et leurs émules britanniques. Dans ces conditions, Pitt envisage en 1799 , sur les bases du rapport du comité secret de la Chambre des communes, de renforcer l'arsenal répressif et de rester inflexible sur la suspension de l'Habeas Corpus. Il est intéressant de constater que ce comité a travaillé non seulement sur l'établissement de listes de sociétés radicales et subversives à proscrire, sur leurs organigrammes - largement surestimés d'ailleurs- mais aussi sur leurs connexions, et sur les couvertures «innocentes» qu'elles emploient pour camoufler leurs agissements. Or, tant le comité que Pitt lui-même ont reçu directement ou par le biais du Home Office des lettres de dénonciation ou de mise en garde contre l'infiltration de loges. Ainsi, John Waring, prêtre catholique à Stonyhurst relate comment un Irlandais du nom de Bernard Kerr lui a dit qu'il était «franc-maçon, chevalier templier et qu'il appartenait à une société populaire qui s'appelait elle-même United Englishmen ». Le 17 avril 1799, l'avocat James Green franc-maçon de Leeds écrit au Home Secretary à propos de la «plus basse classe des francs-maçons " (lower class of freemasons) qu'il accuse d'inclination coupable pour la Révolution française - il évoque le cannibalian government in France. Green indique que ces faux-frères respectent en apparence l'interdiction de toute conversation politique et religieuse en loge, mais que dès la fin des travaux, les agapes sont l'objet de violentes charges contre le gouvernement britannique et d'adhésion au chaos révolutionnaire. Green explique qu'il a gagné l'amitié d'un des membres de la loge qui, sous le sceau du secret maçonnique, 
lui a montré une lettre d'un des chefs des United Irishmen datée de Dublin le 31 mars 1799 et annoncé la tenue d'une assemblée générale en Écosse à Paisley, sous couvert maçonnique. Green propose d'espionner la dite loge et de faire son rapport aux autorités. Peu importe la véracité des faits dénoncés. Ces lettres ont été étudiées par le Comité secret, elles en ont nourri à la fois les inquiétudes et l'argumentaire répressif. On remarquera en outre qu'il ne s'agit pas de dénonciations anonymes.

Le House of Commons secret committee rend son rapport le 15 mars 1799. Il déclare que les documents que lui a soumis le gouvernement présentent les «preuves irréfutables d'une entreprise systématique, mûrie de longue date et orchestrée depuis la France en relation avec des traîtres en Grande-Bretagne, de destruction des lois, de la constitution, du gouvernement, et de toute forme d'ordre civil ou religieux tant dans le royaume de Grande-Bretagne que dans celui d'Irlande, et de dissolution de l'union entre les deux royaumes ». Pour mettre en œuvre cette subversion générale, ont été créées des sociétés politiques d'un type jusqu'alors inconnu au sein du Royaume-Uni et incompatible avec la tranquillité publique. La Chambre des communes entame le débat sur ce rapport, le 19 avril. Pitt insiste sur « la plus importante lutte que nous ayons jamais entreprise contre l'esprit funeste et sans repos du jacobinisme, qui prend de nouvelles formes et dissimule ses objectifs destructeurs et diaboliques sous de nouvelles formes et de nouvelles pratiques». Il poursuit sur la nécessité pour les autorités de s'adapter à cette évolution en intégrant ces nouvelles formes de lutte et de subversion politique. Pitt définit ensuite ce que l'on pourrait appeler les stigmates de la société subversive : membres liés par un engagement illégal de fidélité mutuelle et de secret; secret des procédures de recrutement des membres; direction secrète de la société dont les adhérents ignorent l'existence et les projets criminels. Présenter l'un de ces signes caractéristiques ou "victimaires» (René Girard) doit conduire toute société à être déclarée comme "unlawful confederacy». La loi devrait citer nommément la London Corresponding Society, les United Englishmen, Scotsmen, Irishmen, et Britons. Mais Pitt va plus loin en proposant que soit également considérée comme association ou coalition illégale "toute société dont les membres sont requis de prêter un serment d'engagement", les sociétés devant désormais être astreintes à admettre leurs nouveaux membres lors d'une réunion publique. Pour la franc-maçonnerie, cette extension des critères d'illégalité est catastrophique, puisque le serment maçonnique qui avait été tenu hors du champ de la loi de 1797 est désormais au cœur du débat. En outre, l'organisation d'admission publique des candidats fait disparaitre l'essence même de la franc-maçonnerie comme société à secrets unie par les mystères de l'initiation partagée. Plus encore que lors de la Freimaurerpatent de l'empereur Joseph II en décembre 1785 dans la monarchie des Habsbourg, l'adoption de cette proposition par la loi signifierait la mort de l'ordre.

10 Les Grandes Loges britanniques ont immédiatement compris la gravité de la menace. Le 30 avril 1799, alors même que la proposition de loi passe en deuxième lecture devant la Chambre des communes, Pitt reçoit une demande officielle d'audience de la part de leurs dirigeants. Le 2 mai, une délégation conjointe emmenée par le comte de Moira, Acting Grand Master de la Grande Loge d'Angleterre (Modernes) et par le duc d'Atholl, grand maître de la Grande Loge des Anciens et ancien grand maître de la Grande Loge d'Écosse se rend au 10 Downing Street. Les comptes rendus de la rencontre dans les archives de la Grande Loge des Modernes indiquent que le premier ministre a exprimé la bonne opinion qu'il avait de l'ordre et affirmé son soutien à toute disposition de la loi qui permette d'assurer sa protection et sa liberté d'association, dès lors que des 
mesures appropriées auront été prises pour empêcher les comploteurs d'abriter sous la forme de loges maçonniques innocentes leurs projets criminels. Moira rappelle qu'il s'est déjà spontanément engagé à prévenir le gouvernement de sa majesté et le Parlement de toute intrusion ou camouflage de ce type. Dès la fin de la rencontre, les dignitaires anglais et écossais prennent des mesures d'urgence. Les grands officiers des Anciens se réunissent à la Crown and Anchor Tavern dans le Strand. Ils décident la suspension immédiate de toute procession maçonnique publique et de toutes les assemblées maçonniques particulières, sous quelque motif que ce soit, à l'exception des tenues régulières des loges et chapitres de Royal Arch, ouvertes à tous les maçons visiteurs dont la régularité aura été vérifiée. Il est spécifié que dès la fin des travaux, la loge doit se disperser et tenir fermé son lieu de réunion. Le 6 mai, le duc d'Atholl luimême préside la Grand Lodge of Emergency, qui entérine ces mesures. Ce n'est pas un hasard si la Grande Loge des Anciens est la plus prompte à réduire drastiquement son activité. Dès l'origine, en 1751, elle accueille en effet de nombreux Irlandais, qui en raison de leur origine et de leur extraction sociale nettement inférieure à celle des Modernes, ont vu leur candidature rejetée au seuil des temples. La Grande Loge des Anciens qui a connu un indéniable succès a gardé des liens importants avec la francmaçonnerie irlandaise qui la reconnaît. Le contexte de suspicion généralisée contre les United Irishmen et leurs émules anglais et écossais, l'existence de lettres de dénonciations de la collusion entre loges artisanales et mouvements républicains a clairement poussé le duc d'Atholl à réagir sans délai.

La satisfaction de Pitt est évidente. Convaincu de la volonté et de la capacité des Grandes Loges britanniques à assurer elles-mêmes leur police et la surveillance de leurs membres, il propose lui-même les amendements qui visent à exclure la francmaçonnerie du champ de la loi. Il s'agit clairement d'un système d'autorégulation, qui malgré la tentative avortée du milieu du siècle pour obtenir une charter of incorporation, témoigne de la quasi-reconnaissance institutionnelle des Grandes Loges. C'est d'ailleurs ce point qui concentrera les attaques lorsque le projet de loi sera étudié par la Chambre des Lords: une association qui n'est pas reconnue comme un corps public peut-elle exercer, à la demande de l'État, un pouvoir de police ayant valeur légale ? Pitt propose que les grands secrétaires des obédiences déposent chaque année auprès des juges de paix une déclaration portant la liste de toutes les loges reconnues à travers le pays par leurs grands maîtres respectifs, avec les lieux et dates de tenues. Chaque loge devra tenir un registre où chaque membre déclarera lors de sa réception qu'»il est sincèrement attaché à la constitution, au gouvernement de ce royaume par le roi, les Lords et les Communes, comme la loi l'établit». Ce registre doit être tenu à la disposition et à l'inspection des magistrats locaux. Les Grandes Loges seront officiellement responsables de la police maçonnique, et les loges dont les noms n'apparaîtront pas sur les listes remises par les grands secrétaires seront considérées comme des coalitions criminelles. À ce point des débats, Modernes et Anciens peuvent s'estimer plus que satisfaits : ils ont non seulement écarté la menace d'une mise horsla-loi, mais en se rapprochant de Pitt ils sont devenus des instruments reconnus de l'application de la dite loi.

12 À la Chambre des Lords, le débat est ouvert par le Foreign Secretary, Lord Grenville, c'est-à-dire par un propre membre du gouvernement de Pitt. S'il admet que les loges britanniques ne montrent aucune hostilité envers l'État, Grenville se fait l'écho de la conduite réputée criminelle des sociétés maçonniques dans les pays étrangers. Il considère que les dispositions spécifiques à la franc-maçonnerie prévues dans le projet 
d'amendement constituent une "anomalie juridique»: le Parlement ne peut pas accorder en droit un pouvoir d'auto-régulation aux Grandes Loges sur le fonctionnement desquelles il n'a aucune prise, puisqu'il ne contrôle ni l'élection ni la nomination des officiers. Mais les attaques viennent surtout du comte de Radnor lors de la troisième lecture du projet devant la Chambre des Lords en juin 1799. L'orateur propose un amendement pour supprimer le statut dérogatoire des francs-maçons. Il souligne qu'il n'appartient pas à l'ordre et que, dans les circonstances extraordinaires actuelles, il est sage de ne pas accorder de faveur particulière à la franc-maçonnerie. Il s'appuie sur les Proofs of a Conspiracy du «très érudit Professeur » Robison (1797), qui ont révélé la possible et universelle conspiration des Illuminati, dont la GrandeBretagne n'est pas exempte, et mis l'accent sur leur volonté d'effacer les identités nationales. L'opinion flatteuse de Radnor n'est pas partagée par Adam Ferguson dans sa correspondance avec Alexandre Carlyle où il s'étonne que Robison puisse « traiter de la Maçonnerie sans commencer par expliquer de quoi il s'agit». C'est en tout cas la première référence aux Proofs of a Conspiracy dans les débats parlementaires. Il n'est plus ici question comme au tout début des débats du champ d'application de la loi sur les associations qui inclurait ou non la franc-maçonnerie, de la même façon qu'on discute au même moment de son application aux Inns of Court ou aux sociétés d'astronomie - Pitt craignant qu'elles abritent des sociétés politiques. La francmaçonnerie est visée ès-qualités, pour elle-même, sur la base de révélations antimaçonniques.

Significativement, le duc d'Atholl, membre de la Chambre des Lords, monte à nouveau en première ligne. Comme grand maître des Anciens, il peut attester de la loyauté, de l'innocence de ses frères, du caractère charitable et bienfaisant de leur ordre. Il conteste à un profane, lord Radnor, la possibilité de comprendre l'essence du projet et des vertus maçonniques et balaie les thèses antimaçonniques. Mais, il doit faire face à l'intervention d'un autre franc-maçon, Samuel Horsley, évêque de Rochester, ancien secrétaire de la Royal Society rendu célèbre par son édition des travaux de Newton, qui suggère de distinguer une franc-maçonnerie régulièrement patentée, loyale, innocente et charitable, à laquelle lui-même appartient, de loges sauvages qui pourraient être influencées par les idées subversives venues du continent. S'il comprend comme francmaçon la légitimité du recours au secret, comme législateur, il se dit contraint de le remettre en cause. La situation actuelle ne permet pas de prendre le moindre risque d'intrusion subversive. Or, en raison du secret, mais d'un secret qui peut avoir été détourné de son sens premier, initiatique, rien ne permet de garantir que ces germes révolutionnaires ne sont pas déjà dissimulés dans certains ateliers, ou noyaux maçonniques. Reprenant les réserves de Grenville, Horsley s'oppose à l'autorégulation par les Grandes Loges. Le duc d'Atholl finit alors par accepter le compromis de Grenville pour éviter l'adoption de l'amendement Radnor : les déclarations des loges auprès des juges de paix sont renforcées, précisées et faites directement par leurs secrétaires auprès des juges de paix, sans vérification, filtrage ou quelconque intervention des obédiences. Toute mention des Grandes Loges et de leurs grands secrétaires est supprimée: elles n'ont donc plus de fonction de police reconnue en droit. À ces conditions, les loges ne sont pas considérées comme des sociétés secrètes à caractère subversif. Mais cette exemption ne bénéficie qu'aux loges existantes ; de fait, toute fondation nouvelle est interdite. Après plusieurs lectures par les deux chambres, il apparaît nécessaire de réécrire la loi, qui est finalement adoptée le 12 juillet 1799. 

inoffensif de la franc-maçonnerie, mais la question a fait débat, au sein même du gouvernement, témoignant de la réalité de l'impact en Grande-Bretagne des révélations anti-maçonniques et de la crise sans précédent qu'enregistre alors la franc-maçonnerie continentale, qui transforme radicalement la perception de l'ordre maçonnique pour les deux siècles à venir. La préparation et la discussion de la loi mettent également en perspective historique les débats de la fin des années 1790 .

Si l'on rappelle que la loi de 1799 n'a été abrogée qu'en 1967 - en 1939 un juge de paix du comté d'Essex sommera encore les loges de faire leurs déclarations-, on constate qu'à deux siècles exactement de distance, c'est la même ambiguïté « essentielle » de la sociabilité maçonnique qui ressurgit. Née dans les interstices de la société profane, dans la sphère de l'intime, elle cultive cependant sa position au sein de l'Establishment. Ses protestations d'innocence et ses actions caritatives sont soupçonnées de dissimuler des jeux d'influence et la pratique conjointe de l'entrisme et du favoritisme. Pas plus qu'au $\mathrm{XVIII}^{\mathrm{e}}$ siècle, les apologies maçonniques, les campagnes de communication médiatique visant à ouvrir les temples n'ont pu faire taire les doutes, la suspicion, la curiosité malsaine et les fantasmes. Le secret fait toujours recette, la thèse des supérieurs inconnus qui abusent de la naïveté du gros des frères trouve plus que jamais écho dans l'opinion : Les frères invisibles. Enquêtes sur les dérives de la Franc-maçonnerie de Ghislaine Ottenheimer et Renaud Lecadre ont été plusieurs mois en tête des ventes d'essais-documents (sic) en France en 2001, tandis que les droits de réponse des obédiences sont passés largement inaperçus, comme un plaidoyer pro domo de plus, voire comme la " preuve que, finalement, ils ont bien quelque chose à se reprocher »...

\section{RÉSUMÉS}

L'auteur étudie les étapes de la préparation de la Unlawful Societies Act (1799) qui tente d'étouffer les sociétés politiques britanniques suspectées d'être sous influence française. La francmaçonnerie, également visée par la loi, clame son innocence et manifeste son patriotisme afin d'être épargnée par la nouvelle législation. Si les Grandes Loges sauvent finalement l'essentiel, le débat indique la suspicion qui entoure la franc-maçonnerie en Angleterre et la confusion qui peut régner à la fin des années 1790 entre sociétés secrètes et sociétés à secrets.

English freemasons and the Unlawful Societies Act. The author examines the stages in the preparation of the Unlawful Societies Act (1799) which attempted to stifle British political societies suspected of being under French influence. The law also targeted freemasons who defended themselves through claims of innocence and displays of patriotism. Though the Grand Lodges were able to preserve their essential character, the debates at the time indicate the suspicions which shrouded freemasonry in England and the confusion which reigned at the end of the 1790s between secret societies and secret fraternal orders. 
INDEX

Mots-clés : franc-maçonnerie, Unlawful Societies Act, Pitt

\section{AUTEUR}

\section{PIERRE-YVES BEAUREPAIRE}

Université de Nice Sophia-Antipolis, Parc Valrose, 28, avenue de Valrose, BP 2135, 06103 NICE CEDEX 2 